\title{
Isomorphism on Fuzzy Hypergraphs
}

\author{
C.Radhamani ${ }^{1}$, C.Radhika ${ }^{2}$ \\ ${ }_{1,2}$ (Assistant Professor, Department of Mathematics, Kongu Arts and science college, Erode, Tamil Nadu, India)
}

\begin{abstract}
In this paper, the order,size and degree of the nodes of the isomorphic fuzzyhypergraphs are discussed. Isomorphism between fuzzy hypergraphs is proved to be an equivalance relation.

Keywords - Fuzzy hypergraph, co-weak isomophismr, weak isomorphism, equivalence relation
\end{abstract}

\section{Introduction}

Lofti Zadeh, in his classical paper in 1965 introduced the notion of fuzzy subsets of a set. He also introduced the concept of fuzzy relations. Since then, the fuzzy set theory become a wide research area in various disciplines like medicine, social sciences, engineering, statistics, graph theory, management sciences, computer sciences, artificial intelligence, pattern recognition, expert systems, decision making, robotics, signal processing and automata theory.

Rosenfield introduced in 1975, the concept of fuzzy graphs and the graph theoretic concepts like paths, cycles and connectedness were introduced in fuzzy graphs. In [5] P.Bhattacharya associated a fuzzy graph with a fuzzy graph in the natural way as an automorphism group. The concept of weak isomorphism and isomorphism between fuzzy graphs were introduced by K.R.Bhutani in [4].

Mordeson and Premchand Nair [1] introduced the concept of fuzzy hypergraphs and several fuzzy analogs of hypergraph theory. Operations on fuzzy hypergraphs were introduced by Berge [3] .

In [2] A.Nagoorgani and J.Malarvizhi discussed the isomorphism between fuzzy graphs and some properties of self complementary and self weak complementary fuzzygraphs . properties.

In this paper, we introduce the isomorphism of fuzzy hypergraphs and some of their important

\section{Definition[1]}

\section{Preliminaries}

Let $\mathrm{X}$ be a finite set and let $\varepsilon$ be a finite family of non trivial fuzzy subsets of $\mathrm{X}$ such that $\mathrm{X}=\bigcup_{\mu_{i}} \operatorname{supp} \mu_{i}(x)$. Then the pair $H:(X, \in)$ is called a fuzzy hypergraph and $\in=\left\{E_{1}, E_{2}, \ldots E_{n}\right\}$ is called the collection of edge sets of $H$.

Note

A fuzzy hyper graph $H$ with underlying set $X$ is $H:\left(X, \mu_{i}, \rho\right)$ where $\mu_{i}: X \rightarrow[0,1]$ are fuzzy

subsets, $\rho: \in \rightarrow[0,1]$ is a fuzzy relation on the fuzzy subsets

$\mu_{i}$ such that $\rho\left(\left\{\mathrm{x}_{1}, x_{2}, \ldots \ldots x_{r}\right\}\right) \leq \mu_{\mathrm{i}}\left(x_{1}\right) \times \mu_{i}\left(x_{2}\right) \times \ldots . . \times \mu_{i}\left(x_{r}\right)$

\section{Definition 1}

Given a fuzzy hypergraph $H:(X, \mu, \rho)$ with the underlying set $X$, the order of $H$ is defined and denoted as

$$
p=\sum \wedge \mu_{i}(x)
$$

and size of $H$ is defined and denoted as

$$
q=\sum_{\left\{x_{1}, x_{2} \ldots x_{r}\right\}=E_{I} \subset X} \rho\left(x_{1}, x_{2} \ldots x_{r}\right)=\sum_{E_{i} \subset X} \rho\left(E_{i}\right)
$$

\section{Definition 1}

\section{Isomorphism - Basic Properties}

A homomorphism of fuzzy hypergraphs $h: H \rightarrow H^{\prime}$ is a map $h: X \rightarrow X^{\prime}$ which satisfies

$$
\wedge \mu_{i}(x) \leq \wedge \mu_{i}^{\prime}(h(x)) \forall x \in X \text { and }
$$


Definition 2

$$
\rho\left(x_{1}, x_{2} \ldots . . x_{r}\right) \leq \rho^{\prime}\left(h\left(x_{1}\right), h\left(x_{2}\right), \ldots . h\left(x_{r}\right)\right) \forall\left\{x_{1}, x_{2} \ldots \ldots x_{r}\right\}=E_{i} \subset X
$$

A weak isomorphism $h: H \rightarrow H^{\prime}$ is a map, $h: X \rightarrow X^{\prime}$ which is a bijective homomorphism that satisfies $\wedge \mu_{i}(x)=\wedge \mu_{i}^{\prime}(h(x)) \quad \forall x \in \mathrm{X}$

Example 3.1

Let $H:\left(X, \mu_{i}, \rho\right)$ and $H^{\prime}:\left(X^{\prime}, \mu_{i}^{\prime}, \rho^{\prime}\right)$ be two fuzzy hypergraphs with underlying sets

$X=\{a, b, c, d\}$ and $X=\left\{a^{\prime}, b^{\prime}, c^{\prime}, d^{\prime}\right\}$ where $E_{1}=\{a, b, c\}, E_{2}=\{c, d\}$ are the edges of $H$ and

$E_{1}^{\prime}=\left\{a^{\prime}, c^{\prime}\right\}, E_{2}^{\prime}=\left\{a^{\prime}, b^{\prime}, d^{\prime}\right\}$ are the edges of $H^{\prime}$ defined by

$$
\begin{aligned}
& \rho\left(E_{1}\right)=1 / 5, \rho\left(E_{2}\right)=1 / 6 \\
& E_{1}=\{(a, 1 / 4)(b, 1 / 3)(c, 1 / 5)\} \\
& E_{2}=\{(c, 1 / 4)(d, 1 / 5)\} \\
& \rho^{\prime}\left(E_{1}^{\prime}\right)=1 / 6 \quad \rho^{\prime}\left(E_{2}^{\prime}\right)=1 / 6 \\
& E_{1}^{\prime}=\left\{\left(a^{\prime}, 1 / 4\right)\left(c^{\prime}, 1 / 3\right)\left(d^{\prime}, 1 / 5\right)\right\} \\
& E_{2}^{\prime}=\left\{\left(a^{\prime}, 1 / 2\right)\left(b^{\prime}, 1 / 3\right)\left(c^{\prime}, 1 / 5\right)\right\}
\end{aligned}
$$

Incidence matrices are given as follows:
$E_{1} \quad E_{2}$
a $1 / 4 \quad 0$
b $1 / 3 \quad 0$
c $1 / 51 / 4$
d $0 \quad 1 / 5$

$\begin{array}{ccc} & E_{1}^{\prime} & E_{2}^{\prime} \\ a^{\prime} & 1 / 4 & 1 / 2 \\ b^{\prime} & 0 & 1 / 3 \\ c^{\prime} & 1 / 3 & 1 / 5 \\ d^{\prime} & 1 / 5 & 0\end{array}$

Definition 3

A co- weak isomorphism $h: H \rightarrow H^{\prime}$ is a map $h: X \rightarrow X^{\prime}$ which is a bijective homomorphism that satisfies

$$
\rho\left(x_{1}, x_{2} \ldots . x_{r}\right)=\rho^{\prime}\left(h\left(x_{1}\right), h\left(x_{2}\right), \ldots . h\left(x_{r}\right)\right) \quad \forall\left\{x_{1}, x_{2}, \ldots x_{r}\right\}=E_{i} \subset X
$$

\section{Example 3.2}

Let $H:\left(X, \mu_{i}, \rho\right)$ and $H^{\prime}:\left(X^{\prime}, \mu_{i}^{\prime}, \rho^{\prime}\right)$ be the fuzzy hypergraphs with underlying sets $\mathrm{X}=\{a, b, c, d\}$ and $\mathrm{X}=\left\{a^{\prime}, b^{\prime}, c^{\prime}, d^{\prime}\right\}$

$$
\begin{aligned}
& E_{1}=\{(a, 1 / 4)(b, 1 / 4)(c, 1 / 3)\} \\
& E_{2}=\{(c, 1 / 2)(d, 1 / 3)\} \\
& \rho\left(E_{1}\right)=1 / 5 \quad \rho\left(E_{2}\right)=1 / 6
\end{aligned}
$$


$E_{1}^{\prime}=\left\{\left(a^{\prime}, 1 / 3\right)\left(d^{\prime}, 1 / 3\right)\right\}$

$E_{2}^{\prime}=\left\{\left(a^{\prime}, 1 / 2\right)\left(b^{\prime}, 1 / 3\right)\left(c^{\prime}, 1 / 4\right)\right\}$

$\rho^{\prime}\left(E_{1}^{\prime}\right)=1 / 5 \quad, \quad \rho^{\prime}\left(E_{2}^{\prime}\right)=1 / 6$

Incidence matrices are given as follows

\begin{tabular}{|c|c|c|c|}
\hline & $E_{2}$ & & $E_{1}^{\prime}$ \\
\hline a $1 / 4$ & 0 & $a^{\prime}$ & $1 / 3$ \\
\hline b $\quad 1 / 4$ & 0 & $b^{\prime}$ & 0 \\
\hline $1 / 3$ & $1 / 2$ & $c^{\prime}$ & 0 \\
\hline 0 & $1 / 3$ & $d^{\prime}$ & $1 / 3$ \\
\hline
\end{tabular}

\section{Definition 4}

A isomorphism $h: H \rightarrow H^{\prime}$ is a map $h: X \rightarrow X^{\prime}$ which is a bijective homomorphism that satisfies $\wedge \mu_{i}(x)=\wedge \mu_{i}^{\prime}(h(x)) \forall x \in X$ $\rho\left(x_{1}, x_{2} \ldots . x_{r}\right)=\rho^{\prime}\left(h\left(x_{1}\right), h\left(x_{2}\right), \ldots . h\left(x_{r}\right)\right) \forall\left\{x_{1}, x_{2}, \ldots x_{r}\right\}=E_{i} \subset X$

We denote the isomorphism of the hypergraphs $H$ and $H^{\prime}$ as $H \cong H^{\prime}$

\section{Remark}

1. A weak isomorphism of fuzzy hypergraph preserves the weights of the nodes but not necessarily the weights of the edges.

2. A co- weak isomorphism preserves the weights of the edges but not necessarily the weights of the nodes.

3. An isomorphism preserves both the weights of the edges and the nodes.

4. An endomorphism of a fuzzy hypergraph $H$ is a homomorphism of $H$ to itself.

5. An automorphism of a fuzzy hypergraph $H$ is an isomorphism of $H$ to itself.

6. When the two fuzzy hypergraphs $H$ and $H^{\prime}$ are same the weak isomorphism between them becomes an isomorphism and similarly the co-weak isomorphism between them also becomes isomorphism.

In crisp hyper graphs when two hypergraphs are isomorphic they are of same order. Also the same is true in the case of fuzzy hypergraphs. We prove this result in the following theorem.

Theorem 3.1

For any two isomorphic fuzzy hypergraphs their order and size are same.

Proof

If $h: H \rightarrow H^{\prime}$ is an isomorphism between the fuzzy hyper graphs $H \& H^{\prime}$ with the underlying sets $\mathrm{X} \&$ $\mathrm{X}^{\prime}$ respectively then

$$
\begin{aligned}
& \wedge \mu_{i}(x)=\wedge \mu_{i}^{\prime}(h(x)) \quad \forall x \in \mathrm{X} \\
& \rho\left(x_{1}, x_{2} \ldots . . x_{r}\right)=\rho^{\prime}\left(h\left(x_{1}\right), h\left(x_{2}\right), \ldots h\left(x_{r}\right)\right) \forall\left\{x_{1}, x_{2}, \ldots x_{r}\right\}=E_{i} \subset X
\end{aligned}
$$

$$
\begin{aligned}
& \mathrm{p}=\operatorname{order}(H)=\sum_{x \in X} \wedge \mu_{i}(x)=\sum \wedge \mu_{i}^{\prime}(h(x))=\operatorname{order}\left(H^{\prime}\right) \\
& \mathrm{q}=\operatorname{Size}\left(H^{\prime}\right)=\sum_{\mathrm{E}_{i} \subset \mathrm{X}} \rho\left(\mathrm{E}_{i}\right)=\sum_{\mathrm{E}_{i} \subset \mathrm{X}^{\prime}} \rho^{\prime}\left(\mathrm{E}_{i}^{\prime}\right)=\operatorname{Size}\left(H^{\prime}\right)
\end{aligned}
$$

\section{Corollary}

Converse of the above theorem need not be true. We prove this by an example.

\section{Example 3.3}

Consider the fuzzy graphs $H$ and $H^{\prime}$ with underlying sets $X$ and $X^{\prime}$ as 
$X=\{a, b, c, d\}, X^{\prime}=\left\{a^{\prime}, b^{\prime}, c^{\prime}, d^{\prime}\right\}$ respectively.

Incidence matrices are given as follows

$\begin{array}{lcccccc} & E_{1} & E_{2} & & E_{1}^{\prime} & E_{2}^{\prime} \\ a & 1 / 5 & 1 / 6 & a^{\prime} & 1 / 5 & 1 / 5 \\ b & 0 & 1 / 5 & b^{\prime} & 1 / 6 & 1 / 3 \\ & 1 / 3 & 1 / 5 & c^{\prime} & 1 / 3 & 1 / 5 \\ c & 1 / 2 & 0 & d^{\prime} & 1 / 2 & 0\end{array}$

$\rho\left(E_{1}\right)=1 / 5 \quad, \quad \rho\left(E_{2}\right)=1 / 6 \quad \rho\left(E_{1}^{\prime}\right)=1 / 6, \quad \rho\left(E_{2}^{\prime}\right)=1 / 5$

$p=1 / 6+1 / 5+1 / 5+1 / 2 \quad p^{\prime}=1 / 5+1 / 6+1 / 5+1 / 2$

$\mathrm{q}=1 / 5+1 / 6 \quad q^{\prime}=1 / 6+1 / 5$

Here order and size are same but $H$ is not isomorphic to $H^{\prime}$

Remark

If the fuzzy hypergraphs are weak isomorphic then their orders are same. But the fuzzy hyper graphs of same order need not be weak isomorphic. We prove this by the following example.

\section{Example 3.4}

Consider the fuzzy hypergraphs $H$ and $H^{\prime}$ with underlying sets $X$ and $X^{\prime}$ as $X=\{a, b, c, d\}$ and $X^{\prime}=\left\{a^{\prime}, b^{\prime}, c^{\prime}, d^{\prime}\right\}$ respectively.

Incidence matrices are given as follows

$\begin{array}{ccc} & E_{1} & E_{2} \\ a & 1 / 5 & 1 / 7 \\ b & 0 & 1 / 5 \\ c & 1 / 3 & 1 / 6 \\ d & 1 / 2 & 0\end{array}$

Order $=1 / 7+1 / 5+1 / 6+1 / 2$

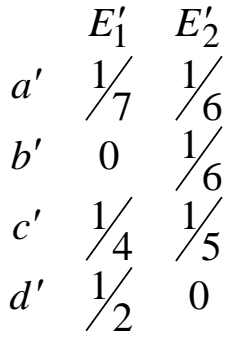

Order $=1 / 7+1 / 6+1 / 5+1 / 2$

Here the order is same. But they are not weak isomorphic.

\section{Remark}

If the fuzzy hypergraphs are co-weak isomorphic, their sizes are same. But the fuzzy hyper graphs of same size need not be co-weak isomorphic. We prove this by the following example.

\section{Example 3.5}

Incidence matrices are given as follows

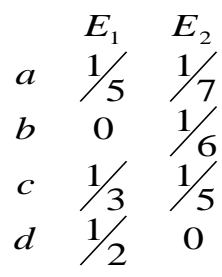

$\begin{array}{ccc} & E_{1}^{\prime} & E_{2}^{\prime} \\ a^{\prime} & 0 & 1 / 5 \\ b^{\prime} & 1 / 7 & 1 / 4 \\ c^{\prime} & 1 / 2 & 1 / 5 \\ d^{\prime} & 1 / 3 & 0\end{array}$




$$
\begin{array}{ll}
\rho\left(E_{1}\right)=1 / 5, & \rho\left(E_{2}\right)=1 / 7 \\
\rho\left(E_{1}^{\prime}\right)=1 / 7, & \rho\left(E_{2}^{\prime}\right)=1 / 5
\end{array}
$$

Size $H=1 / 5+1 / 7 \quad$ Size $\left(H^{\prime}\right)=1 / 7+1 / 5$

Here size of $H$ and $H^{\prime}$ are same. But they are not co-weak isomorphic.

Definition 5

Let $H:\left(X, \mu_{i}, \rho\right)$ be a fuzzy hypergraph. The degree of a vertex is defined as

$d\left(x_{1}\right)=\sum \rho\left(x_{1}, x_{2}, \ldots . x_{r}\right)$ for $x_{1} \neq x_{2} \ldots \ldots \neq x_{r}$

Theorem 3.2

If $H$ and $H^{\prime}$ are isomorphic fuzzy hypergraphs then the degrees of their nodes are preserved.

Proof: Let $h: X \rightarrow X^{\prime}$ be an isomorphism of fuzzy hypergraphs $H$ onto $H^{\prime}$. By the definition of isomorphism

$$
\begin{aligned}
& \rho\left(x_{1}, x_{2} \ldots . x_{r}\right)=\rho^{\prime}\left(h\left(x_{1}\right), h\left(x_{2}\right), \ldots . h\left(x_{r}\right)\right) \quad \forall\left\{x_{1}, x_{2}, \ldots x_{r}\right\}=E_{i} \subset X \\
& \begin{aligned}
d\left(x_{i}\right) & =\sum \rho\left(x_{1}, x_{2}, \ldots . . x_{r}\right) \quad x_{i} \neq x_{r} \\
& =\sum \rho^{\prime}\left(h\left(x_{1}\right), h\left(x_{2}\right), \ldots . h\left(x_{r}\right)\right) \\
& =d\left[h\left(x_{i}\right)\right]
\end{aligned}
\end{aligned}
$$

Corollary 3.17

Converse of the above theorem need not be true. Consider the following incidency matrices.

$\begin{array}{lll} & E_{1} & E_{2} \\ a & 1 / 2 & 1 / 3 \\ & 1 / 4 & 1 / 5\end{array}$

$$
\begin{array}{lll} 
& E_{1}^{\prime} & E_{2}^{\prime} \\
a^{\prime} & 1 / 3 & 1 / 2 \\
& 1 / 3 & 1 / 4 \\
b^{\prime} & 1 / 5 & 1 / 4
\end{array}
$$

$$
\begin{array}{rr}
d(a)=1 / 2+1 / 3 & d\left(a^{\prime}\right)=1 / 3+1 / 2 \\
d(b)=1 / 4+1 / 5 & d\left(b^{\prime}\right)=1 / 5+1 / 4 \\
\rho\left(E_{1}\right)=1 / 4 & \rho^{\prime}\left(E_{1}^{\prime}\right)=1 / 5 \\
\rho\left(E_{2}\right)=1 / 5 & \rho^{\prime}\left(E_{2}^{\prime}\right)=1 / 4
\end{array}
$$

Here degrees of the nodes are preserved but edges are not preserved.

\section{Remark}

The degree of a vertex is measured only by adding the weights of the edges incident with that vertex. But fuzzy hypergraphs preserving the degree of the vertices need not be co-weak isomorphic. 


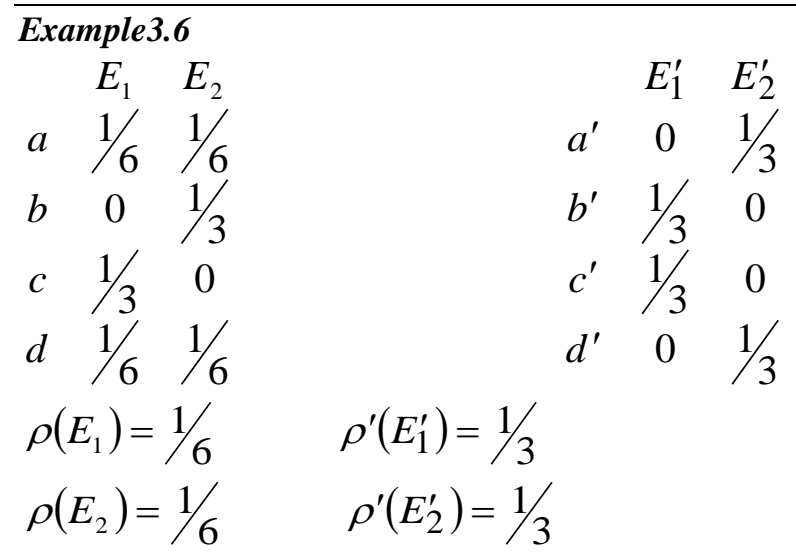

In the above two hypergraphs each vertex is of degree $1 / 3$. But those two hypergraphs are neither co-weak nor weak isomorphic hypergraphs.

\section{Theorem 3.3}

Isomorphism between fuzzy hypergraphs is an equivalence relation.

\section{Proof}

Let $H:\left(X, \mu_{i}, \rho\right), H^{\prime}:\left(X^{\prime}, \mu_{i}^{\prime}, \rho^{\prime}\right), H^{\prime \prime}:\left(X^{\prime \prime}, \mu_{i}^{\prime \prime}, \rho^{\prime \prime}\right)$ be fuzzy hypergraphs with underlying sets $X, X^{\prime}$ and $X^{\prime \prime}$ respectively.

(i) Reflexive:

Consider the identity map $h: X \rightarrow X, h(x)=x \quad \forall x \in X$

This $h$ is a bijective map satisfying.

$\wedge \mu_{i}(x)=\wedge \mu_{i}^{\prime}(h(x)) \quad \forall x \in X \quad$ and
$\rho\left(x_{1}, x_{2} \ldots . . x_{r}\right)=\rho\left(h\left(x_{1}\right), h\left(x_{2}\right), \ldots h\left(x_{r}\right)\right) \quad \forall\left\{x_{1}, x_{2}, \ldots x_{r}\right\}=E_{i} \subset X$

Hence $h$ is an isomorphism of the fuzzy hypergraph to itself. Therefore it satisfies reflexive relation.

(ii) Symmetric:

Let $h: X \rightarrow X^{\prime}$ be an isomorphism of $H$ and $H^{\prime}$, then $h$ is a bijective map $h(x)=x^{\prime} \quad \forall x \in X$

Then $h$ is a bijective map satisfying

$$
\begin{aligned}
\wedge \mu_{i}(x) & =\wedge \mu_{i}^{\prime}(h(x)) \quad \forall x \in X \text { and } \\
\rho\left(x_{1}, x_{2} \ldots . . x_{r}\right) & =\rho^{\prime}\left(h\left(x_{1}\right), h\left(x_{2}\right), \ldots h\left(x_{r}\right)\right) \quad \forall\left\{x_{1}, x_{2}, \ldots x_{r}\right\}=E_{i} \subset X
\end{aligned}
$$

Since $h$ is bijective by (1),

$$
\begin{gathered}
h^{-1}\left(x^{\prime}\right)=x \quad \forall x^{\prime} \in X^{\prime} \\
\wedge\left[\mu_{i}\left(h^{-1}\left(x^{1}\right)\right)\right]=\wedge \mu_{i}^{\prime}\left(x^{\prime}\right) \quad \forall x^{\prime} \in X^{\prime} \quad \text { and } \\
\rho\left(h^{-1}\left(x_{1}^{\prime}\right), h^{-1}\left(x_{2}^{\prime}\right), \ldots . h^{-1}\left(x_{r}^{\prime}\right)\right)=\rho^{\prime}\left(x_{1}, x_{2^{\prime}}^{\prime}, x_{r}^{\prime}\right) \forall\left\{x_{1}, x_{2}, \ldots x_{r}\right\}=E_{i}^{\prime} \subset X
\end{gathered}
$$

Hence we get a 1-1, onto map $h^{-1}: X^{\prime} \rightarrow X$ which is an isomorphism from $H^{\prime}$ to $H$.

$$
H \cong H^{\prime} \Rightarrow H^{\prime} \cong H
$$

(iii) Transitive: 
Let $h: X \rightarrow X^{\prime}$ and $\mathrm{g}: X^{\prime} \rightarrow X^{\prime \prime}$ be an isomorphism of fuzzy hypergraphs $H$ onto $H^{\prime}$ and $H^{\prime}$ onto $H^{\prime \prime}$ respectively.

Then $\mathrm{g} \circ \mathrm{h}$ is a 1-1, onto map from $X \rightarrow X^{\prime \prime}$ where $(g \circ h)(x)=g(h(x)) \quad \forall x \in X$

As $h: \mathrm{X} \rightarrow \mathrm{X}^{\prime}$ is an isomorphism,

$h(x)=x^{\prime}, \forall x \in X$

$\wedge \mu_{i}(x)=\wedge \mu_{i}^{\prime}(h(x)) \quad \forall x \in X$

$\rho\left(x_{1}, x_{2} \ldots . . x_{r}\right)=\rho^{\prime}\left(h\left(x_{1}\right), h\left(x_{2}\right), \ldots . h\left(x_{r}\right)\right) \forall\left\{x_{1}, x_{2}, \ldots . x_{r}\right\}=E_{i} \subset X$

$\wedge\left(\mu_{i}(x)=\wedge \mu_{i}^{\prime}\left(x^{\prime}\right)\right) \quad \forall x \in X$

$\rho\left(x_{1}, x_{2} \ldots . x_{r}\right)=\rho^{\prime}\left(x_{1}^{\prime}, x_{2}^{\prime} \ldots . x_{r}^{\prime}\right) \quad \forall\left\{x_{1}, x_{2}, \ldots x_{r}\right\}=E_{i} \subset X$

As $g: X^{\prime} \rightarrow X^{\prime \prime}$ is an isomorphism

$g\left(x^{\prime}\right)=x^{\prime \prime}, \forall x^{\prime} \in X^{\prime}$

$\wedge\left(\mu_{i}^{\prime}\left(x^{\prime}\right)\right)=\wedge\left(\mu_{i}^{\prime \prime}\left(g\left(x^{\prime}\right)\right)\right) \quad \forall x^{\prime} \in X^{\prime}$

$\rho^{\prime}\left(x_{1}^{\prime}, x_{2}^{\prime} \ldots . . x_{r}^{\prime}\right)=\rho^{\prime \prime}\left(g\left(x_{1}^{\prime}\right), g\left(x_{2}^{\prime}\right), \ldots . g\left(x_{r}^{\prime}\right)\right) \quad \forall\left\{x_{1}^{\prime}, x_{2}^{\prime}, \ldots x_{r}^{\prime}\right\}=E_{i}^{\prime} \subset X^{\prime}$

From (4) and (6) and using

$$
\begin{aligned}
h(x)=x^{\prime}, & \forall x \in X \\
\wedge\left(\mu_{i}(x)\right)= & \wedge\left(\mu_{i}^{\prime}\left(x^{\prime}\right)\right)=\wedge\left(\mu_{i}^{\prime \prime}\left(g\left(x^{\prime}\right)\right)\right) \quad \forall x^{\prime} \in X^{\prime} \\
= & \wedge\left(\mu_{i}^{\prime \prime} g(h(x))\right) \quad \forall x \in X
\end{aligned}
$$

From (5) and (7),

$$
\begin{aligned}
\rho\left(x_{1}, x_{2} \ldots . . x_{r}\right) & =\rho^{\prime}\left(x_{1}^{\prime}, x_{2}^{\prime} \ldots . x_{r}^{\prime}\right) \quad \forall\left\{x_{1}, x_{2}, \ldots x_{r}\right\}=E_{i} \subset X \\
& =\rho^{\prime \prime}\left(g\left(x_{1}^{\prime}\right), g\left(x_{2}^{\prime}\right), \ldots g\left(x_{r}^{\prime}\right)\right) \quad \forall\left\{x_{1}^{\prime}, x_{2}^{\prime}, \ldots x_{r}^{\prime}\right\}=E_{i}^{\prime} \subset X^{\prime} \\
& =\rho^{\prime \prime}\left(g\left(h\left(x_{1}\right)\right), g\left(h\left(x_{2}\right)\right), \ldots g\left(h\left(x_{r}\right)\right)\right) \quad \forall\left\{x_{1}, x_{2}, \ldots x_{r}\right\} \subset X
\end{aligned}
$$

Therefore $\mathrm{g} \circ \mathrm{h}$ is an isomorphism between $H$ and $H^{\prime \prime}$.

Hence isomorphism between fuzzy hypergraphs is an equivalence relation.

\section{Theorem 3.4}

Weak isomorphism between fuzzy hypergraphs satisfies the partial order relation.

Proof:

Let $H:\left(X, \mu_{i}, \rho\right) H^{\prime}:\left(X^{\prime}, \mu_{i}^{\prime}, \rho^{\prime}\right), H^{\prime \prime}:\left(X^{\prime \prime}, \mu_{i}^{\prime \prime}, \rho^{\prime \prime}\right)$ be fuzzy hypergraphs with underlying sets $X$, $X^{\prime}, X^{\prime \prime}$ respectively.

(i) Reflexive:

Consider the identity map $h: X \rightarrow X$ such that $h(x)=x$, for all $x \in X$.

This $\mathrm{h}$ is a bijective map satisfying

$$
\begin{aligned}
\wedge \mu_{i}(x) & =\wedge \mu_{i}^{\prime}(h(x)) \quad \forall x \in X \\
\rho\left(x_{1}, x_{2} \ldots . . x_{r}\right) & \leq \rho\left(h\left(x_{1}\right), h\left(x_{2}\right), \ldots . h\left(x_{r}\right)\right) \quad \forall\left\{x_{1}, x_{2}, \ldots x_{r}\right\}=E_{i} \subset X
\end{aligned}
$$

Hence $\mathrm{h}$ is a weak isomorphism of the fuzzy hypergraph to itself. Therefore $\mathrm{H}$ is weak isomorphic to itself.

(ii) Anti symmetric:

Let $\mathrm{h}$ be a weak isomorphism between $H$ and $H^{\prime}$ and $\mathrm{g}$ be a weak isomorphism between $H^{\prime}$ and $H$. i.e. $h$ : $X \rightarrow X^{\prime}$ is a bijective map $h(x)=x^{\prime}$, satisfying

$$
\wedge \mu_{i}(x)=\wedge \mu_{i}^{\prime}(h(x)) \quad \forall x \in X \quad \text { and }
$$


$\rho\left(x_{1}, x_{2} \ldots . . x_{r}\right) \leq \rho^{\prime}\left(h\left(x_{1}\right), h\left(x_{2}\right), \ldots . h\left(x_{r}\right)\right) \quad \forall\left\{x_{1}, x_{2}, \ldots x_{r}\right\}=E_{i} \subset X$

$g: \mathrm{X}^{\prime} \rightarrow \mathrm{X}$ is a bijective map satisfying

$$
\begin{aligned}
& g\left(x^{\prime}\right)=x, \forall x^{\prime} \in X^{\prime} \text { satisfying } \\
& \wedge \mu_{i}(x)=\wedge \mu_{i}^{\prime}\left(g\left(x^{\prime}\right)\right) \forall x^{\prime} \in X^{\prime} \\
& \rho^{\prime}\left(x_{1}^{\prime}, x_{2}^{\prime} \ldots \ldots x_{r}^{\prime}\right) \leq \rho\left(g\left(x_{1}^{\prime}\right), g\left(x_{2}^{\prime}\right), \ldots g\left(x_{r}^{\prime}\right)\right) \quad \forall\left\{x_{1}^{\prime}, x_{2}^{\prime}, \ldots x_{r}^{\prime}\right\}=E_{i}^{\prime} \subset X^{\prime}
\end{aligned}
$$

The inequalities (8) and (9) holds good on the finite sets $X$ and $X^{\prime}$ only when $H$ and $H^{\prime}$ have the same number of edges and the corresponding edges have same weight. Hence $H$ and $H^{\prime}$ are identical.

(iii) Transitive: Let $h: X \rightarrow X^{\prime}$ and $g: X^{\prime} \rightarrow X^{\prime \prime}$ be weak isomorphisms of the fuzzy hypergraphs $X$ onto $X^{\prime}$ and $X^{\prime}$ onto $X^{\prime \prime}$ respectively.

Then $\mathrm{g} \circ \mathrm{h}$ is a $1-1$ onto map from $X$ to $X^{\prime \prime}$ where

$(g \circ h)(x)=g(h(x)) \quad \forall x \in X$

Given $\mathrm{h}$ is a weak isomorphism, $h(x)=x^{\prime} \quad \forall x \in X$

$\wedge \mu_{i}(x)=\wedge \mu_{i}^{\prime}(h(x)) \quad \forall x \in X$

$\rho\left(x_{1}, x_{2} \ldots . x_{r}\right) \leq \rho\left(h\left(x_{1}\right), h\left(x_{2}\right), \ldots . h\left(x_{r}\right)\right) \forall\left\{x_{1}, x_{2}, \ldots x_{r}\right\}=E_{i} \subset X$

Similarly as $\mathrm{g}$ is a weak isomorphism from $X^{\prime}$ to $X$ " we have,

$g\left(x^{\prime}\right)=x^{\prime \prime}, \quad x^{\prime} \in \mathrm{X}^{\prime}$

$\wedge\left(\mu_{i}^{\prime}\left(x^{\prime}\right)=\left(\mu_{i}^{\prime \prime}\left(g\left(x^{\prime}\right)\right)\right)\right) \quad \forall x^{\prime} \in X^{\prime}$

$\rho^{\prime}\left(x_{1}^{\prime}, x_{2}^{\prime} \ldots . x_{r}^{\prime}\right) \leq \rho^{\prime \prime}\left(g\left(x_{1}^{\prime}\right), g\left(x_{2}^{\prime}\right), \ldots . g\left(x_{r}^{\prime}\right)\right) \forall\left\{x_{1}^{\prime}, x_{2}^{\prime}, \ldots x_{r}^{\prime}\right\}=E_{i}^{\prime} \subset X^{\prime}$

From the above, we have

$$
\begin{gathered}
\wedge\left(\mu_{i}(x)=\wedge \mu_{i}^{\prime}\left(x^{\prime}\right)\right) \quad \forall x \in X \\
=\wedge \mu_{i}^{\prime \prime} g\left(x^{\prime}\right) \quad \forall x^{\prime} \in X^{\prime} \\
=\wedge \mu_{i}^{\prime \prime} g\left(h\left(x^{\prime}\right)\right) \quad \forall x \in X
\end{gathered}
$$

From (10),(11),(12)\&(13)

$$
\begin{aligned}
\rho\left(x_{1}, x_{2} \ldots \ldots x_{r}\right) & \leq \rho^{\prime}\left(x_{1}^{\prime}, x_{2}^{\prime} \ldots \ldots x_{r}^{\prime}\right) \quad \forall\left\{x_{1}, x_{2}, \ldots x_{r}\right\}=E_{i} \subset X \\
& \leq \rho^{\prime \prime}\left(g\left(x_{1}^{\prime}\right), g\left(x_{2}^{\prime}\right), \ldots . g\left(x_{r}^{\prime}\right)\right) \quad \forall\left\{x_{1}^{\prime}, x_{2}^{\prime}, \ldots x_{r}^{\prime}\right\}=E_{i}^{\prime} \subset X^{\prime} \\
& =\rho^{\prime \prime}\left(g\left(h\left(x_{1}\right)\right), g\left(h\left(x_{2}\right)\right), \ldots . g\left(h\left(x_{r}\right)\right)\right) \forall\left\{x_{1}, x_{2}, \ldots x_{r}\right\} \subset X
\end{aligned}
$$

Therefore $\mathrm{g} \circ \mathrm{h}$ is a weak isomorphism between $H$ and $H^{\prime \prime}$ i.e. weak isomorphism satisfies transitivity. Hence weak isomorphism between fuzzy hyper graphs is a partial order relation.

\section{Conclusion}

In this paper isomorphism between fuzzy hypergraphs is proved to be an equivalence relation and weak isomorphism is proved to be a partial order relation. Similarly it is expected that co-weak isomorphism can also be proved to be a partial order relation.

\section{References}

[1]. Moderson, J.N, and P .S. Nair Fuzzy Graphs and Fuzzy Hypergraphs Physica verlag ,Heidelberg 1998 ; Second Edition 2001.

[2]. A.Nagoorgani and J.Malarvizhi ., Isomorphism on Fuzzy Graphs.

[3]. C.Berge., Graphes et Hypergraphes. Dunod ,Paris,(Graphs and Hypergraphs, North-Holland,Amsterdam, 1973, revised translation) 1970

[4]. Bhutani, K.R., On Automorphism of Fuzzy graphs, Pattern Recognition Letter 9: 159-162,1989.

[5]. Bhattacharya, P, Some Remarks on fuzzy graphs, pattern Recognition Letter 6: 297-302, 1987. 